\title{
SPHERES WITH LOCALLY PINCHED METRICS
}

\author{
ROBERT J. CURRIER
}

(Communicated by Jonathan M. Rosenberg)

\begin{abstract}
The purpose of this paper is the construction of metrics on spheres whose curvatures are locally almost constant but which have large variation globally. This construction also applies to spherical space forms.
\end{abstract}

The purpose of this paper is the construction of metrics on spheres whose curvatures are locally almost constant but which have large variation globally. To make this idea precise, if $\delta$ is a positive number not greater than 1, we say that a metric is locally $\delta$-pinched if, at each point, the maximum and minimum sectional curvatures satisfy the inequality $\delta \leq K_{\min }(p) / K_{\max }(p)$. All manifolds considered here are positively curved, so we take the statement that $M$ is locally $\delta$-pinched to include the assertion that $M$ is positively curved. A classical result of Schur may be phrased: All locally 1-pinched manifolds have globally constant curvature. In contrast with Schur's result, Gribkov [G] has constructed metrics on Euclidean 3-space that are locally $\delta$-pinched, for any $\delta<1$, but whose global variation of curvature is large. This paper provides the first compact examples with this curvature behavior.

An important result in local pinching, due to Ruh [R], is that for $\delta$ sufficiently close to 1 a compact locally $\delta$-pinched manifold is diffeomorphic to a spherical space form. The proof proceeds by showing that the local average of the scalar curvature is almost constant. The examples given here demonstrate the effectiveness of this technique. More recently, Huisken [H], Margerin [M], and Nishikawa $[\mathrm{N}]$ have used evolution equations for the metric to estimate the local pinching required.

By contrast, Seaman [S] has shown that compact, orientable, even-dimensional manifolds whose second Betti number is nonzero and which are locally $\frac{1}{4}$-pinched are isometric to a constant multiple of the canonical metric on $\mathbf{C} P^{n}$; thus they are globally $\frac{1}{4}$-pinched.

Properties of the examples are summarized in the following proposition:

Received by the editors June 16, 1988, and, in revised form, November 28, 1988.

1980 Mathematics Subject Classification (1985 Revision). Primary 53C20.

Key words and phrases. Pinching, spherical space forms. 
Proposition. On every sphere $S^{n}, n \geq 3$, for every $\delta, \frac{1}{2} \leq \delta<1$ and for every $M>1$, there exists a smooth metric satisfying the following conditions:

(i) the metric is locally $\delta$-pinched;

(ii) the global minimum of the curvature satisfies $K_{\min } \leq 1$; the global maximum of the curvature satisfies $K_{\max } \geq M$;

(iii) the diameter $d$ satisfies the inequality $\pi \leq d \leq 4$.

Construction. These metrics are constructed as warped products $S^{n-1} \times_{f} I$ of the standard sphere with an interval $I=[-a, a]$. The metric is given as $g=d t^{2}+f(t)^{2} g_{0}$, where $t$ is the coordinate in $I, g_{0}$ is the canonical metric on $S^{n-1}$, and $f(t)$ is to be determined, subject to the condition that $f( \pm a)=0$ to give the appropriate identifications. The sectional curvature of the spheres $S^{n-1} \times\{t\}$, which we denote $K_{\tan }(t)$, is given by

$$
K_{\tan }(t)=\frac{1-f^{\prime}(t)^{2}}{f(t)^{2}}
$$

and the sectional curvature of 2-planes tangent to the $t$-curves, which we denote $K_{\mathrm{rad}}(t)$, is given by

$$
K_{\mathrm{rad}}(t)=-\frac{f^{\prime \prime}(t)}{f(t)} .
$$

These are the extreme values of the curvature on a given sphere $S^{n-1} \times\{t\}$, so we guarantee local pinching by requiring that these curvatures be related: $K_{\mathrm{rad}}(t)=p(t) K_{\tan }(t)$, where $p(t)$ is a smooth function taking values in the interval $[\delta, 1]$.

Writing the last equation in terms of $f$, we have:

$$
f^{\prime \prime}(t)=-\frac{p(t)\left[1-f^{\prime}(t)^{2}\right]}{f(t)} .
$$

In any region where $f(t)$ is bounded away from zero, this differential equation has a unique solution with given initial values. We choose initial conditions $f(0)=1$ and $f^{\prime}(0)=0$, which give $K_{\tan }(0)=1$ and $K_{\mathrm{rad}}(0)=p(0)$. The lines $f(t)= \pm t+k$ are solutions of $(1)$ with $\left|f^{\prime}(t)\right|=1$, so solutions that have $\left|f^{\prime}(0)\right| \neq 1$ have $\left|f^{\prime}(t)\right| \neq 1$ for all $t$ where $f(t)$ is positive. Consequently, $f^{\prime \prime}(t)$ is negative for all $t$ where $f(t)>0$, so $f(t)$ is concave and nonconstant; hence $f(t)$ is positive on some finite interval, $I$.

We now assume that the pinching function $p(t)$ is chosen so that it depends on $t$ via the value of $f(t)$; i.e. $p(t)=p(f(t))$. Then $f(-t)$ is also a solution of (1) with the given initial conditions; by uniqueness of solutions, we determine that $f$ is even, so the interval on which $f$ is positive is symmetric, $I=(-a, a)$. We now choose $p(f(t))$ so that the metric is smooth at $t= \pm a$ and has the required global variation of curvature. Given $\delta$ and $M$, we choose an $\varepsilon$ so that $0<2 \varepsilon<M^{(2 \delta-2)^{-1}}$. Defining $p(y)$ to be a $C^{\infty}$ function whose range is contained in $[\delta, 1]$ and for which $p(y)=\delta$ if $y \geq 2 \varepsilon$ while $p(y)=1$ if $y \leq \varepsilon$, 
then equation (1) can be integrated to give the inverse function $t=f^{-1}(y)$ for $0 \leq t \leq a, 0 \leq y \leq 1$; namely,

$$
t=\int_{y}^{1}\left[1-\exp \left(-\int_{u}^{1} \frac{2 p(v)}{v} d v\right)\right]^{-1 / 2} d u
$$

The inequality $\frac{1}{2} \leq \delta \leq p(y) \leq 1$ yields $\pi / 2 \leq f^{-1}(0) \leq 2$, so the diameter is between $\pi$ and 4 . Since $p(y)$ is constant for $y \geq 2 \varepsilon$, we find that, for $f(t) \geq 2 \varepsilon$, the curvature is given by $K_{\tan }(t)=\frac{1-f^{\prime}(t)^{2}}{f(t)^{2}}=f(t)^{2 \delta-2}$. In particular, for $f(t)=M^{(2 \delta-2)^{-1}}$, we have $K_{\tan }(t)=M$. Since $p(y)=1$ for $y \leq \varepsilon$, the regions near $t= \pm a$ are isometric to spherical caps of constant curvature; therefore the metric is smooth where $f(t)=0$.

Remark. These metrics are symmetric under the antipodal map of the sphere $(x, t) \rightarrow(-x,-t)$, so there are induced metrics on $\mathbf{R} P^{n}, n \geq 3$, that are locally $\delta$-pinched and have large global variation of curvature. In the case of other spherical space forms, $S^{n} / \Gamma$, it is possible to modify the metric on $S^{n}$ within small balls centered at the points of one $\Gamma$-orbit in $S^{n}$ to obtain $\Gamma$ equivariant metrics on $S^{n}$ that are locally $\delta$-pinched and have large curvature variation. These metrics descend to $S^{n} / \Gamma$ with the same curvature behavior.

\section{ACKNOWLEDGMENT}

I would like to thank the Department of Mathematics of the University of Pennsylvania for their hospitality during my visit in the spring of 1988 .

\section{REFERENCES}

[G] J. V. Gribkov, The incorrectness of Schur's theorem, Soviet Math. Dokl. 21 (1980), 922-925.

[H] G. Huisken, Ricci deformation of the metric on a Riemannian manifold, J. Differential Geom. 21 (1985), 47-62.

[M] C. Margerin, Pointwise pinched manifolds are space forms, Proc. Sympos. Pure Math. 44 (1986), 307-328.

[N] S. Nishikawa, Deformation of Riemannian metrics and manifolds with bounded curvature ratios, Proc. Sympos. Pure Math. 44 (1986), 343-352.

[R] E. Ruh, Riemannian manifolds with bounded curvature ratios, J. Differential Geom. 17 (1982), 643-653.

[S] W. Seaman, Existence and uniqueness of algebraic curvature tensors with prescribed properties and an application to the Sphere theorem, preprint.

Department of Mathematics, Smith College, Northampton, Massachusetts 01063 